\title{
On the modelling of thermal radiation at the top surface of a vacuum arc remelting ingot
}

\author{
P.-O. Delzant ${ }^{1,2}$, B. Baqué ${ }^{1}$, P. Chapelle ${ }^{1}$, A. Jardy ${ }^{1}$
}

\author{
${ }^{1}$ Institut Jean Lamour - UMR 7198 CNRS/Université de Lorraine, \\ Laboratory of Excellence DAMAS, Campus ARTEM, 2 allée André Guinier, \\ 54000 Nancy Cedex, France \\ ${ }^{2}$ TIMET Savoie, Avenue Paul Girod, 73400 Ugine, France
}

Keywords: Vacuum Arc Remelting, Thermal radiation, Radiosity method Corresponding author: P. Chapelle pierre.chapelle@ univ-lorraine.fr

\begin{abstract}
Two models have been implemented for calculating the thermal radiation emitted at the ingot top in the VAR process, namely a crude model that considers only radiative heat transfer between the free surface and electrode tip and a more detailed model that describes all radiative exchanges between the ingot, electrode and crucible wall using a radiosity method. From the results of the second model, it is found that the radiative heat flux at the ingot top may depend heavily on the arc gap length and the electrode radius, but remains almost unaffected by variations of the electrode height. Both radiation models have been integrated into a CFD numerical code that simulates the growth and solidification of a VAR ingot. The simulation of a Ti-6-4 alloy melt shows that use of the detailed radiation model leads to some significant modification of the simulation results as compared to the simple model. This is especially true during the hot-topping phase, where the top radiation plays an increasingly important role as compared to the arc energy input. Thus, whereas the crude model has the advantage of its simplicity, use of the detailed model should be preferred.
\end{abstract}




\section{Introduction}

32

Vacuum Arc Remelting (VAR) is a metallurgical process used for the production of high quality ingots of special steel or nickel-based superalloy. It is also the final stage in the melting cycle of reactive metals such as titanium and zirconium alloys. A detailed understanding of the VAR process is of prime importance because of the strategic role and high market value of those alloys ${ }^{1}$. The VAR process, as illustrated in Figure 1, involves melting a consumable metallic electrode under a high vacuum to form a secondary ingot with a good structural quality. Melting of the electrode is assured by an electric arc maintained between the electrode tip (acting as the cathode) and the top of the ingot (acting as the anode). The liquid metal drops formed at the electrode tip fall through the arc plasma and progressively build up the ingot, which solidifies in contact with the walls of a water-cooled copper crucible. This ingot is composed of three parts: the liquid pool, the fully solidified metal and the intermediate mushy zone. The electric arc can be confined by an axial magnetic field created by an external induction coil in order to stabilize the arc. This magnetic field is also used to stir the liquid metal, in order to enhance the chemical homogeneity of the ingot. 


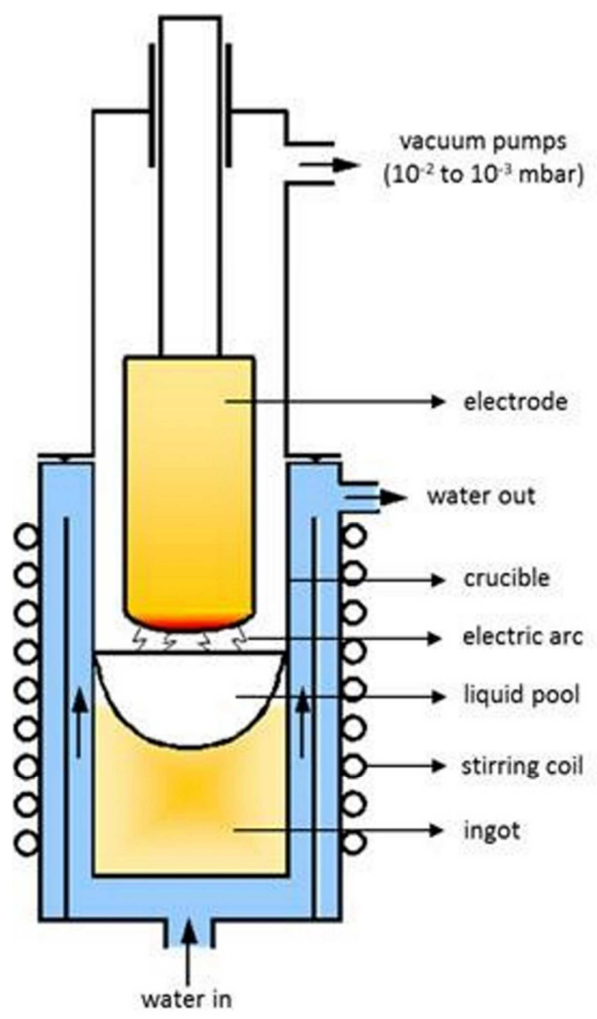

Figure 1: VAR process schematic representation.

The energy flux at the ingot top plays a key role in the process, as it has a direct effect on the temperature and velocity fields in the molten metal, which both determine the local solidification conditions. The total energy flux at the ingot top may be divided into three main contributions: the heat stored in the incoming drops, the energy input from the arc plasma, and the net radiative energy flux at the ingot top surface. In the present paper, we will concentrate on this latter contribution. Note that the radiative energy flux is expected to be much smaller than the two other input energy fluxes during most of the melt, yet its contribution may become more important during the hot-topping stage at the end of the melt, which is performed with a reduced arc power.

One of the classical approaches adopted to study the VAR process is based on the development of a mathematical CFD model that describes the ingot growth and solidification 
during a VAR operation. Several such models have been described in the literature. They involve solving the conservation equations of mass, momentum and energy, while accounting for turbulence and electromagnetic effects in the molten pool as well as the solidification of the metal. The majority of the models consider a 2D axisymmetric geometry. One of those model is SOLAR ${ }^{2}$. Another one is the model MeltFlow-VAR ${ }^{3}$. However, with the aim of better taking into account the precise distribution and possible motion of the arc, Pericleous et al. ${ }^{4}$ have recently proposed a model based on a 3D geometry. Moreover, their macromodel was coupled to a microscale simulation of the solidification process.

As far as the description of the ingot top thermal radiation is concerned, the only detailed work dealing with that subject we are aware of is due to Ballantyne ${ }^{5}$ who represented the radiative heat transfer in a VAR furnace using an electrical network analogy based on the radiosity method. This method takes into account the radiative heat exchanges between all surfaces elements inside the furnace. The radiation model was combined with simplified ingot and electrode models, allowing the prediction of the melt rate and liquid pool depth values, which were found to be in good agreement with measurements 5 .

In the present paper, we describe our model for evaluating the ingot top radiation similar to that derived by Ballantyne ${ }^{5}$. Our proposed model is then incorporated into a CFD macromodel developed at Nancy, that allows computation of the growth and solidification of a VAR ingot. Then we compare the thermal and velocity fields inside the ingot predicted by that model with results obtained using a crude one, based on a simplified description of the radiative heat exchanges. 
In the radiative heat transfer models presented below, two main hypotheses are made. The first hypothesis is that the arc plasma in the interelectrode region is transparent to thermal radiation. There are two main reasons why we decided to consider that the arc is transparent to thermal radiation. The volume fraction of macroparticles in the plasma (mainly tiny liquid metal droplets ejected at the cathode surface) is extremely low, so the absorption of radiation within the arc is probably not important. In addition, it is widely accepted in the vacuum arc literature that (at low current density levels similar to those employed in a VAR furnace) the power radiated by the arc represents a negligible fraction of the total arc power ${ }^{6}$ hence radiation from the plasma may be neglected. More detailed data on the radiative properties of the electric arc in VAR would be needed for a more refined model. It is interesting to mention that Ballantyne ${ }^{7}$ found that the arc cannot be considered as opaque. The second assumption is that all surfaces are considered as grey and diffusive surfaces. Thus, their emissivity $\varepsilon$ depends only on the surface material and the material state (solid or liquid). Two approaches for calculating the flux radiated from the ingot top surface are described below. The first approach is a simplified model that does not account for the fill ratio, hence directly uses the Stefan-Boltzmann law. The second approach is a more detailed model based on the radiosity method.

\section{II.1 Simplified model: Stefan-Boltzmann law}

In this model, the net energy flux density radiated by each surface element $\mathrm{i}$ of the ingot top $M_{i}^{\text {net }}$ (in $\mathrm{W} / \mathrm{m}^{2}$ ) is simply calculated from the Stefan-Boltzmann law ${ }^{8}$ :

$$
M_{i}^{\text {net }}=\epsilon_{i} \sigma\left(T_{i}^{4}-T_{e l e c}^{4}\right)
$$

where $\sigma=5.67 \times 10^{-8} \mathrm{~W} \cdot \mathrm{m}^{-2} \cdot K^{-4}$ is the Stefan-Boltzmann constant, $T_{i}$ is the temperature of surface $i$ and $T_{\text {elec }}$ is the temperature of the liquid film covering the electrode tip. This approach in fact considers that the electrode diameter is identical to that of the ingot (fill ratio 
equal to 1) and neglects the contributions of the crucible and the annular gap between the crucible and electrode in the radiative exchanges. Because the ingot top is assumed to radiate in an environment at a higher temperature than in reality, the radiative heat fluxes and thus the energy loss of the ingot will be underestimated.

\section{II.2 Advanced model: Radiosity method}

In this model inspired by the work of Ballantyne ${ }^{6}$, the flux radiated from the ingot top surface is calculated considering all radiative exchanges inside the furnace, i.e. taking into account the radiation between the ingot top surface, the tip and lateral surface of the electrode and the crucible wall. This requires calculating the radiative heat fluxes from each of these surfaces. The model described here uses the radiosity method ${ }^{7}$.

The radiosity represents the radiation flux density leaving a given surface $\mathrm{i}$ and is defined (for an opaque surface) as the sum of the emitted and reflected radiation flux densities:

$$
J_{i}=\varepsilon_{i} \sigma T_{i}^{4}+\left(1-\varepsilon_{i}\right) \sum_{j=1}^{n} F_{i, j} J_{j}
$$

In this equation, the reflected flux density has been written as a function of geometrical view factors, $F_{i, j}$. The view factor $F_{i, j}$ between the surfaces $i$ and $j$ is the proportion of the total radiative flux leaving surface $i$ that reaches $j$. One should mention three useful relationships between view factors. View factors obey a reciprocity relationship $S_{i} F_{i, j}=S_{j} F_{j, i}$, where $S_{i}$ and $S_{j}$ are the areas of surfaces $i$ and $j$. The sum of the view factors from a given surface is unity (known as the summation rule): $\sum_{j} F_{i, j}=1$. Yet another useful relation comes from the superposition rule, by which the view factor to a surface is, at large scale, the sum of the view factors to the parts of that surface: $F_{i, j+k}=F_{i, j}+F_{i, k}$. Note that view factors are tabulated in the literature for many specific configurations. For complex configurations, view factors may 
be determined using a Monte-Carlo ray tracing method. For a system of $\mathrm{n}$ surfaces forming an enclosure, one obtains from (2) a system of $\mathrm{n}$ linear algebraic equations for the determination of the $\mathrm{n}$ unknown radiosities. Once the radiosities have been determined, the net energy flux density radiated by a surface element $i$ of the ingot top may be calculated from the following relation:

$$
M_{i}^{\text {net }}=\frac{\varepsilon_{i}}{1-\varepsilon_{i}}\left(\sigma T_{i}^{4}-J_{i}\right)
$$

Note that in the above procedure, the surface temperature must be given for each surface.

Figure 2 presents the geometrical configuration considered in our model, detailing every surface involved for the calculation of the ingot top thermal radiation. Surfaces 1 and 3 describe the crucible wall. As it will appear below, the crucible wall was divided into two surfaces to make the calculation of some of the view factors easier. Surface 2 represents the bottom surface of the electrode. Along the lateral surface of the electrode, two regions are distinguished. The first region (ver) corresponds to the lower part of the electrode, where a strong vertical variation of the temperature takes place. The second region (surface $f 0$ ) corresponds to the upper part of the electrode, where the temperature can be considered as uniform. Note that region ver is subdivided into several surface elements, in order to better take into account the temperature variation in this region. The ingot top surface is divided into three regions. The first region (cen) corresponds to the portion of the top surface, which does not see the surface $f 2$ (see Figure 2). The second region (int) corresponds to the portion of the top surface, which is able to see the surface $f 2$ but not the lateral surface of the electrode. Finally, the third region (ext) corresponds to the aiming portion of the top surface (in the annulus zone), which is able to see all other surfaces of the domain. Similarly to region ver, each of these three regions is subdivided into several surface elements of uniform temperature in order to take into consideration the temperature variation over the ingot top surface. 
Finally, surfaces $f 1$ and $f 2$ are both virtual surfaces that do not radiate any heat. Surface $f 1$ is introduced to make easier the calculation of several view factors. Surface $f 2$ is employed to close the domain.

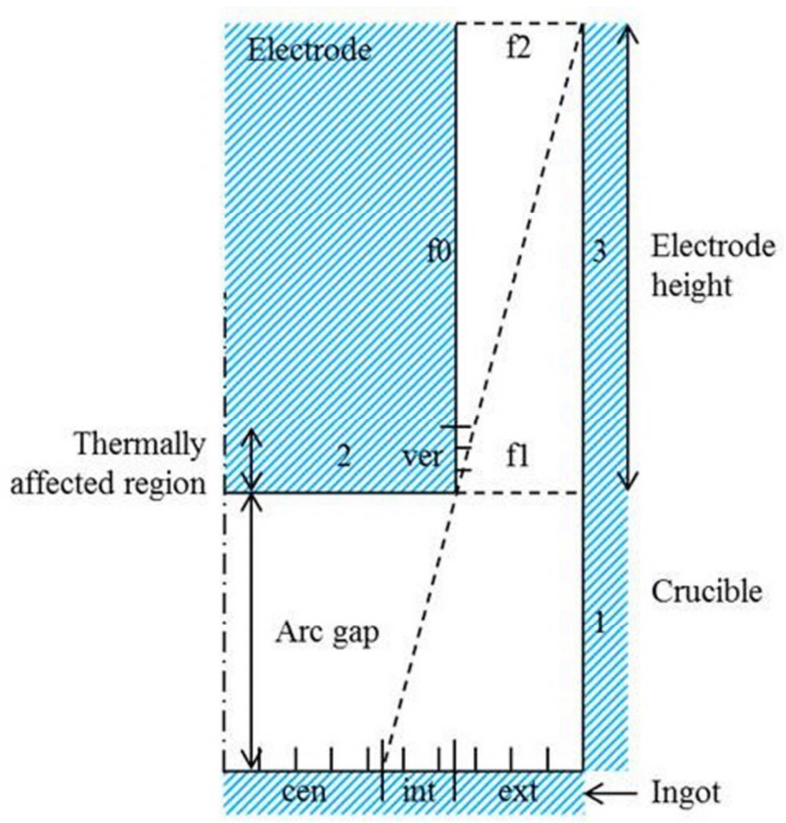

Figure 2: Top furnace schematic representation in the radiation model.

Almost all the view factors involved in our model may be computed using formula tabulated in the literature. The view factors tabulated formula used in this model can be found in the publication of Leuenberger and Person ${ }^{9}$ for the view factors between disks, rings and surfaces of a cylindrical geometry, Buschmann and Pittman ${ }^{10}$ for the view factors between surfaces of sections of cylinder and Brockmann ${ }^{11}$ for the view factors between surfaces of two concentric cylinders. However, for a few complex view factors, no formulas are available and their evaluation relies on some approximations which are detailed below. This concerns in particular pairs of surfaces for which the sight of view is partially occulted by the electrode.

\section{Calculation of $F_{f 2,1}$}


175 The evaluation of the view factor $F_{f 2,1}$ is based on the superposition principle:

$$
F_{f 2,1}=F_{f 2,1+3}-F_{f 2,3}
$$

The view factor between surface $\mathrm{f} 2$ and the combination of surfaces 1 and 3 is calculated from a tabulated formula by considering that the electrode is an infinite cylinder. Note that such an approach is valid as long as the surface $f 2$ does not see the surface 1 through the interelectrode gap, i.e. as long as the height of the electrode is much larger than the interelectrode gap. The view factor $F_{f 2,3}$ in (4) is calculated using a tabulated formula without any assumption regarding the height of the electrode.

Calculation of $F_{\text {int }_{i}, f 2}$ and $F_{\text {ext }}, f 2$

The view factor between any surface $h\left(h=\left\{i n t_{i}\right.\right.$, ext $\left.\left._{i}\right\}\right)$ at the ingot top and the surface $f 2$ is calculated using the following approximation:

$$
F_{h, f 2}=F_{h, 3+f 2} \times F_{f 1, f 2} / F_{f 1,3+f 2} \quad h=\left\{\text { int }_{i}, \text { ext }_{i}\right\}
$$

This amounts considering that the ratio of the solid angle between surfaces $h$ and $f 2$ over the solid angle between $h$ and $(3+f 2)$ is identical to the ratio of the solid angle between $f 1$ and $f 2$ over the solid angle between $f 1$ and $(3+f 2)$. This equation is an approximation for calculating view factor $F_{h, f 2}$. In (5), the view factors $F_{f 1, f 2}$ and $F_{f 1,3+f 2}$ are obtained from tabulated formula, whereas the view factor $F_{h, 3+f 2}$ is calculated by:

$$
F_{h, 3+f 2}=1-\sum_{k \notin\{3 ; f 2 ; f 1\}} F_{h, k} \quad h=\left\{\text { int }_{i}, \text { ext }_{i}\right\}
$$

\section{Calculation of $F_{1,3}, F_{\text {int }}, 3$ and $F_{\text {ext }}, 3$}

The determination of the view factors $F_{h, 3}\left(h=\left\{1, i n t_{i}\right.\right.$, ext $\left.\left._{i}\right\}\right)$ is based on the summation rule: 


$$
F_{h, 3}=1-\sum_{k \notin\{3 ; f 1\}} F_{h, k} \quad h=\left\{1, \text { int }_{i}, \text { ext }_{i}\right\}
$$

195

196

197

198

199

200

201

202

203

204

205

206

207

208

209

210

211

212

Because of the approximations made when evaluating $F_{h, f 2}\left(h=\left\{1, i n t_{i}, e x t_{i}\right\}\right)$, the values of $F_{h, 3}\left(h=\left\{1\right.\right.$, int $_{i}$, ext $\left.\left._{i}\right\}\right)$ obtained by this relation should be considered as approximated values.

\section{Verification}

In this section, our intention is to determine and discuss the influence of the assumptions made in the model described above when evaluating the non-tabulated view factors. For this purpose, exact values of these view factors have been determined using the Monte-Carlo ray tracing method ${ }^{12}$.

This method consists of several steps. The first step is to select randomly the coordinates $(R$, $\alpha$ ) of the starting position of a ray on the emitting surface. In the second step, the emission direction of the ray is defined by selecting randomly a couple of angles $(\theta, \varphi)$. The third step is to track the trajectory of the emitted ray and determine the first surface it will intercept. All steps are repeated for a very large number of rays, so that the sample size is statistically significant. Finally, the view factor between the emitting surface and a given surface corresponds to the ratio of the number of times that surface was hit to the total number of rays emitted. The parameters $R, \alpha, \theta$ and $\varphi$ are defined as follows:

$$
\begin{gathered}
R=r_{i}+\left(r_{e}-r_{i}\right) * \sqrt{n_{1}} \\
\alpha=2 \pi * n_{2} \\
\theta=2 \pi * n_{3} \\
\varphi=\sin ^{-1}\left(\sqrt{n_{4}}\right)
\end{gathered}
$$


where $n_{i}$ are independent random numbers between 0 and 1 and $r_{i}$ (resp. $r_{e}$ ) is the inner (resp. outer) radius of the annular shape emitting surface $\left(r_{i}=r_{e}\left(\right.\right.$ resp. $\left.r_{i}=0\right)$ in the case of cylindrical (resp. disk) shape surfaces).

Before using the Monte-Carlo ray tracing method to determine the approximated view factors, the correct implementation of the method was checked by applying the method to calculate known tabulated view factors, namely the view factors between surface elements at the ingot top and the electrode bottom surface (surface 2). Figure 3 compares the values of the view factor obtained with the tabulated formula with those computed with the Monte-Carlo method (considering $10^{8}$ rays for each surface).

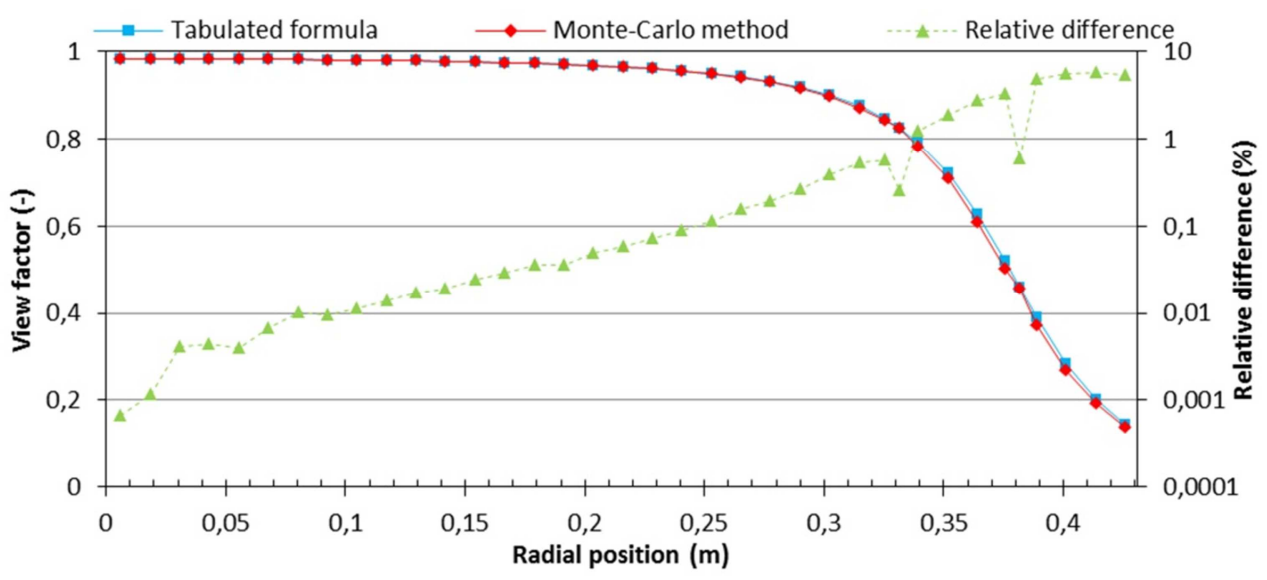

Figure 3: View factor between a surface element at the ingot top and the bottom surface of the electrode. Comparison between results obtained with tabulated formulas and results determined with the Monte-Carlo method. Calculations performed for an ingot radius of $0.432 \mathrm{~m}$, an electrode radius of $0.381 \mathrm{~m}$ and an arc gap of $0.05 \mathrm{~m}$.

Figure 3 shows that both methods give very similar values of the view factors, with a relative difference between the two results less than a few percent, which may be attributed to the statistical nature of the Monte-Carlo results. Quite logically, the increase in the relative difference with the radial position is related to the decrease of the view factor. So, the good 
agreement between both calculation methods validates our implementation of the MonteCarlo ray tracing method.

Having checked that the Monte-Carlo method is correctly implemented, we then apply the method to determine the view factors, whose evaluation in section II is based on approximations (Eqs. (4) to (7)).

Considering first the view factors $F_{1,3}$ and $F_{f 2,1}$, we recall that an approximation is used to estimate those view factors only when the electrode height is smaller than the arc gap. Table 1 compares for such conditions the values of those view factors obtained using the proposed approximations with their values obtained using the Monte-Carlo method. The approximated view factors exhibit a significant error. Yet, as it will be shown later, due to the small absolute values of those view factors, the error induced by the approximations has a very low impact on the evaluation of the flux radiated at the ingot top, which is the parameter of main interest in the present study.

In Figure 4, the values of the view factors between surface elements at the ingot top and respectively surfaces $f 2$ and 3 calculated using the approximations described in section II are compared to the values obtained with the Monte-Carlo method. First it should be pointed out that the results given by our model for surface elements located in the central region (cen) (which extends up to $0.3759 \mathrm{~m}$ ) are naturally in very good agreement with the Monte-Carlo results, since our model employs a tabulated formula in this region. In the intermediate and external regions, we observe in contrast that the approximated view factors are slightly different from the Monte-Carlo view factors. Moreover, Figure 4 shows a trend to larger discrepancies when one approaches the crucible wall. 


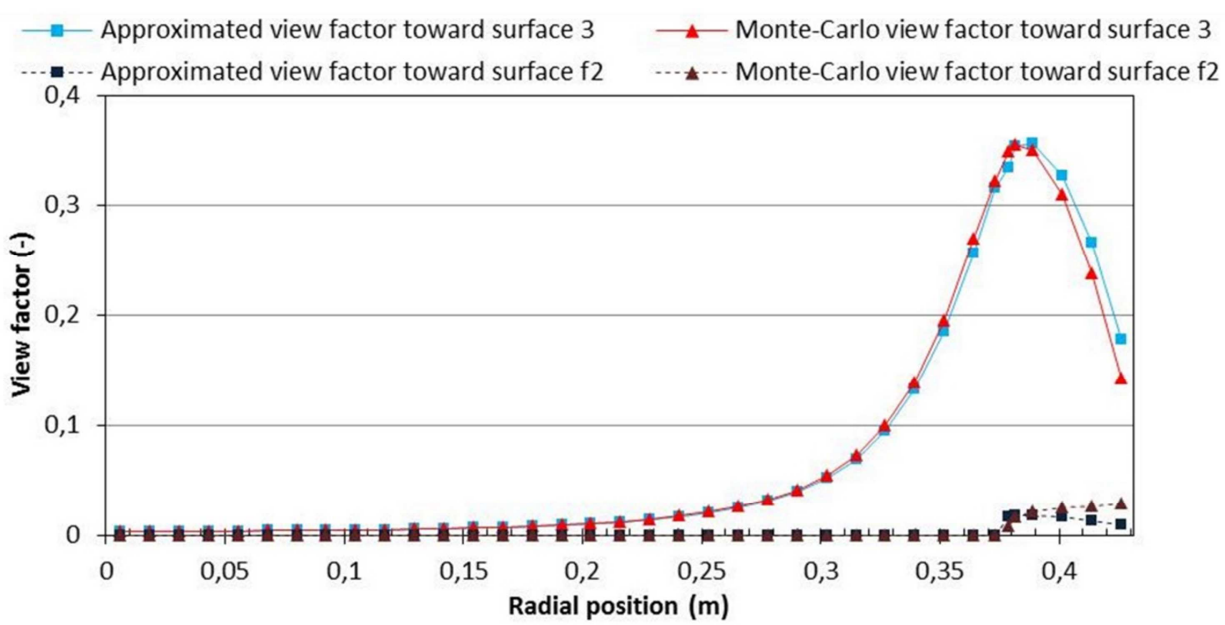

Figure 4: View factor from surface elements of the ingot top toward surface 3 and surface $f 2$ obtained using the approximate method and the Monte-Carlo method. Calculations performed for an ingot radius of $0.432 \mathrm{~m}$, an electrode radius of $0.381 \mathrm{~m}$, an electrode height of $0.5 \mathrm{~m}$ and an arc gap of $0.05 \mathrm{~m}$.

Finally, the ability of our model to compute with a good accuracy the heat flux density radiated from every location at the ingot top, despite the approximations made when evaluating the non-tabulated view factors, is tested. Our model predictions are compared with a result determined with the non-tabulated view factors being obtained from the Monte-Carlo method. Figure 5 presents the relative difference between the two results for various conditions relative to the end (short electrode) of a VAR melt. The emissivity values and the temperature of every surface of the domain considered in those calculations are given respectively in Table 2 and in Table 3. The thermophysical properties of Ti-6-4 alloy required for the simulation were taken from Valencia and Quested ${ }^{13}$ except for the emissivity which is taken from Boivineau et al. ${ }^{14}$. 


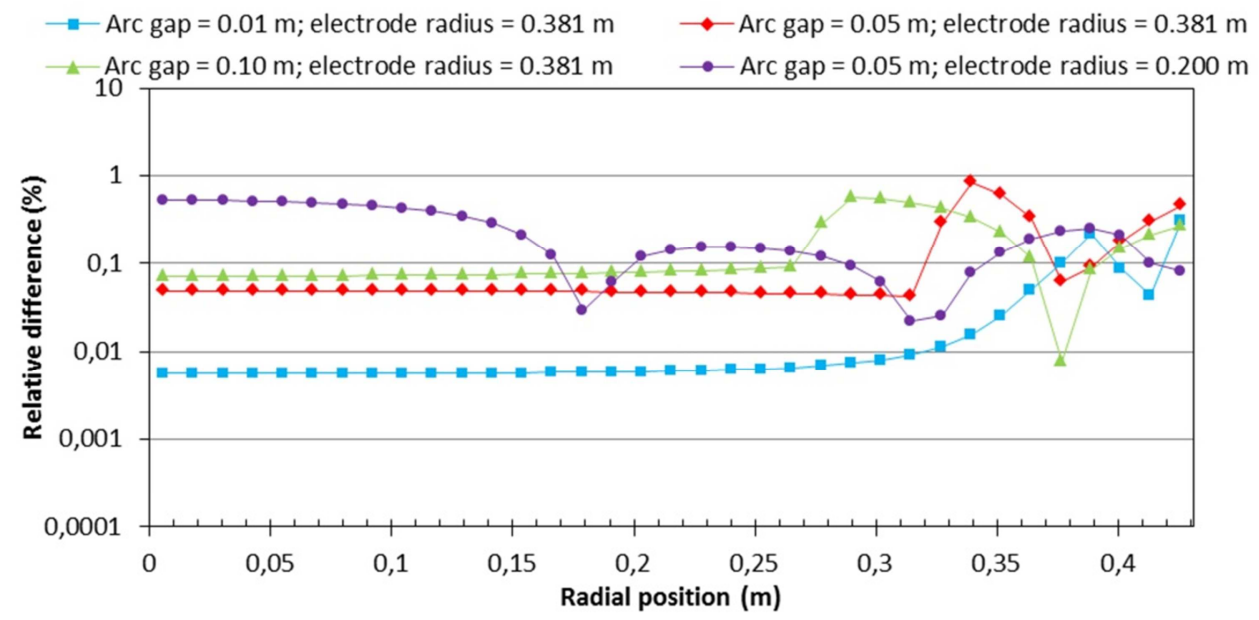

Figure 5: Relative difference in the heat flux density radiated at the ingot top depending on the calculation method (approximate and Monte-Carlo methods) of the nontabulated view factors for an electrode height of $0.05 \mathrm{~m}$.

Figure 5 shows that the approximations made when evaluating the non-tabulated view factors have a negligible impact on the radiative heat flux density computed at the ingot top for all tested configurations. This result may be explained by the fact that the approximations concern view factors toward a surface which either has a low temperature (surface 3) as compared to that of the ingot top or does not emit any radiation (surface $f 2$ ). Therefore, the radiative fluxes originating from these surfaces have a negligible influence on the computation of the radiation emitted at the ingot top. The only case where the approximations could have an impact would be when the temperature of the ingot top is of the same order of magnitude as that of surface 3 . This could occur during the cooling phase of the ingot after extinction of the arc. Finally, it must be noted that the Monte-Carlo ray tracing method has a high computational cost. Because of this cost and given the negligible impact on the ingot top radiation of a precise calculation of the non-tabulated view factors using the Monte-Carlo method, we decided hereafter to evaluate those non-tabulated view factors not by the MonteCarlo method but using the approximations described in Section II. 
This section is separated into two parts. The first part is a parametric study, aimed at analysing the influence of various geometric parameters on the heat flux density radiated at the ingot top. In the second part, we describe the integration of the two proposed radiation models (i.e. the simplified and advanced models) in the CFD software SOLAR ${ }^{2}$ which simulates the growth and solidification of a VAR ingot. Then we compare the ingot simulation results obtained with the two models.

\section{IV.1 Impact of the geometric parameters on the ingot top radiation}

In this section, we apply the detailed radiation model to calculate the radial profile of the heat flux density radiated at the ingot top and we investigate the influence of various geometric parameters on this profile. The model is applied to an electrode and an ingot made of Ti-6-4 alloy, whose emissivity is given in Table 2, and the surface temperatures are given in Table 3. The temperature at the ingot top surface is considered to be uniform. All calculations have been performed with a mesh of 35 surfaces at the ingot top and 10 surfaces in the region ver along the lateral surface of the electrode. The influences of a variation of the arc gap, electrode radius and electrode height on the profile of the heat flux density radiated at the ingot top are illustrated in Figures 6 to 8.

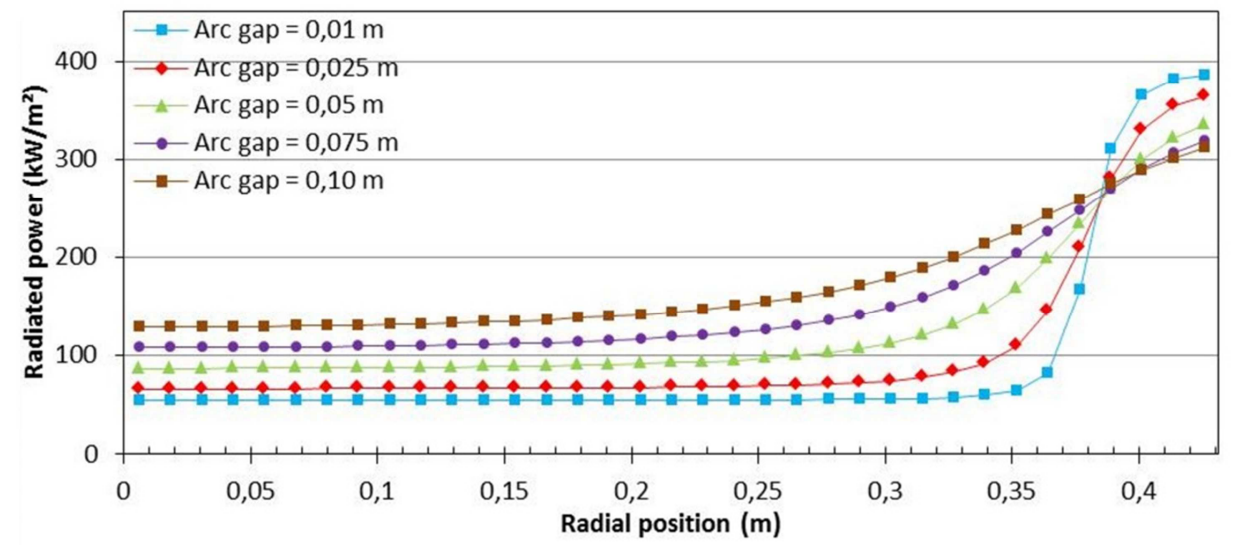



radius of $0.432 \mathrm{~m}$, an electrode radius of $0.381 \mathrm{~m}$ and an electrode height of $1.5 \mathrm{~m}$.

It is observed that the ingot top radiation depends heavily on the arc gap and the electrode radius. The smaller the arc gap, the larger the difference between the radiative flux density emitted by ingot surface elements located underneath the electrode and that emitted by ingot surface elements located below the annular gap between the electrode and crucible. This can be explained as follows. In the case of short arc gaps, the ingot surface elements located underneath the electrode see mainly the tip of the electrode (surface 2) which has a temperature similar to that of the ingot, yielding small radiative heat flux densities. By contrast, the ingot surface elements located below the annular gap see mainly surfaces which have a low temperature, which implies larger radiative heat flux densities. When the arc gap increases, the fraction of the solid angle of the ingot surface elements located underneath the electrode which is occupied by the "cold" surfaces increases, leading to an increase in the located below the annular gap which is occupied by the tip of the electrode will also increase, leading to a decrease of the radiation.

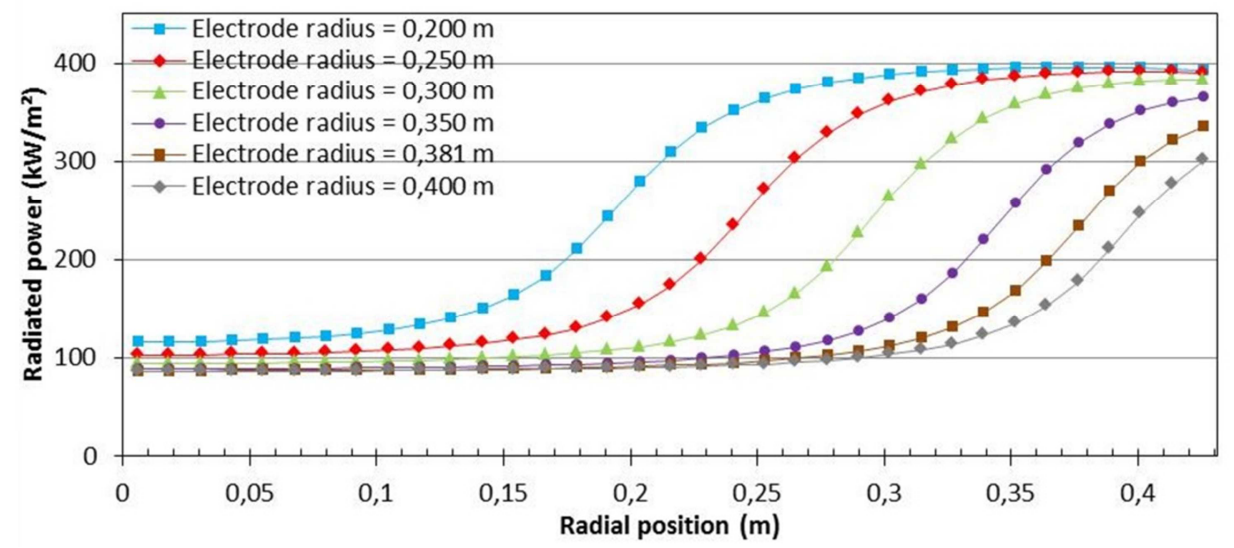
ingot radius of $0.432 \mathrm{~m}$, an electrode height of $1.5 \mathrm{~m}$ and an arc gap of $0.05 \mathrm{~m}$. 
As it was expected, a variation of the electrode radius strongly affects the location of the transition between the low radiation region below the electrode and the high radiation region below the annular gap. Again, this may be explained considering the proportion of the solid angle of the ingot surface elements located underneath the electrode which is occupied by the "hot" surface of the electrode (surface 2). The greater the electrode radius, the larger the number of surface elements seeing mainly surface 2 , which results in an increase of the low radiation region at the ingot top. This explains the apparition of an isosbestic point on figure 6 at approximatively $0.38 \mathrm{~m}$, which is closed to the constant electrode radius.

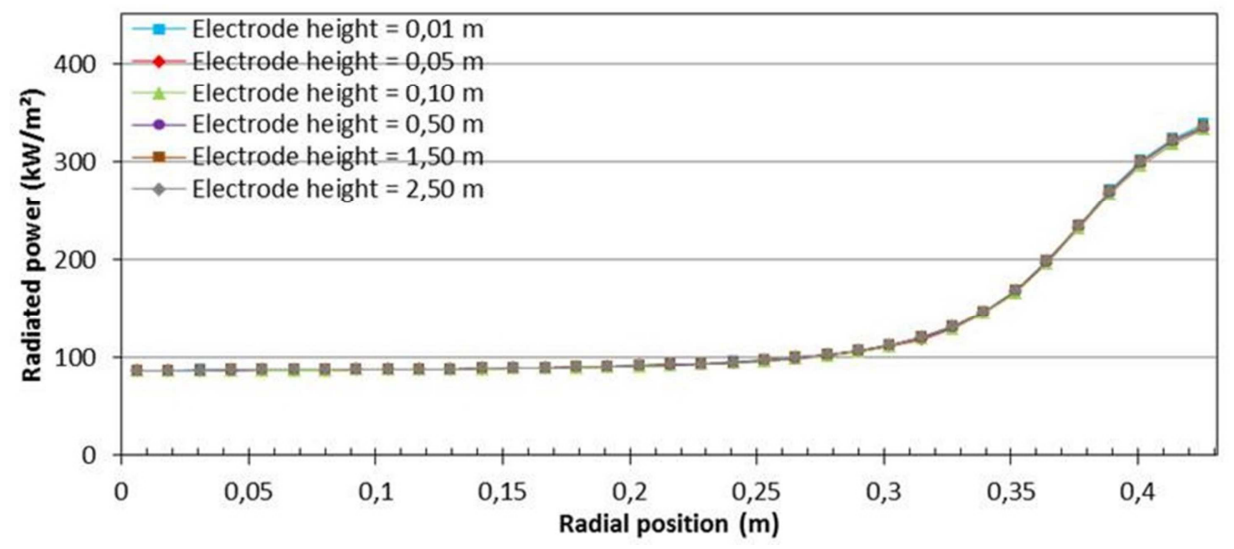

Figure 8: Ingot top radiation for different electrode heights. Calculations performed for an ingot radius of $0.432 \mathrm{~m}$, an electrode radius of $0.381 \mathrm{~m}$ and an arc gap of $0.05 \mathrm{~m}$.

In contrast to the variations in the arc gap and electrode radius, a change of the electrode height has a negligible influence on the ingot top radiation. The height of the electrode impacts essentially the proportion of the flux radiated by the ingot toward surfaces 3 , f0 and f2, and the surfaces composing the region ver. Except for the latter, those surfaces have similar low temperature. Therefore, the heat flux density radiated at the ingot top is only little affected by a variation in the electrode height. 
The two radiation models described in section II have been integrated into the CFD macromodel SOLAR ${ }^{15}$. In this section, we simulate the melt of a Ti-6-4 electrode and compare the results obtained using both radiation models.

The operating parameters of the simulated melt are as follows. The melt consists of two main sequences. The first main sequence (seq. 1) lasts 181.2 min. During this sequence, the arc current is set to $33 \mathrm{kA}$, the voltage is set to $44.7 \mathrm{~V}$ and the melt rate is set to $27 \mathrm{~kg} / \mathrm{min}$. Note that these values are taken from the work of Patel et al. ${ }^{16}$. Before this sequence, the arc current, arc voltage and melt rate were set to $6.5 \mathrm{kA}, 28.5 \mathrm{~V}$ and $0 \mathrm{~kg} / \mathrm{min}$ during $6.8 \mathrm{~min}$ then increased progressively during $2 \mathrm{~min}$ from these values to the ones of the first main melt sequence. The second sequence (seq. 2), which corresponds to the hot-topping stage, lasts 60 min. During this sequence, the arc current is set to $6 \mathrm{kA}$, the arc voltage to $20 \mathrm{~V}$ and the melt rate to zero. Between sequence 1 and sequence 2, the arc current, voltage and melt rate are progressively decreased during $10 \mathrm{~min}$. Figure 9 presents the time evolution of those parameters. During all the melt, a constant unidirectional stirring with a magnetic field of 1.4 $\mathrm{mT}$ is applied.

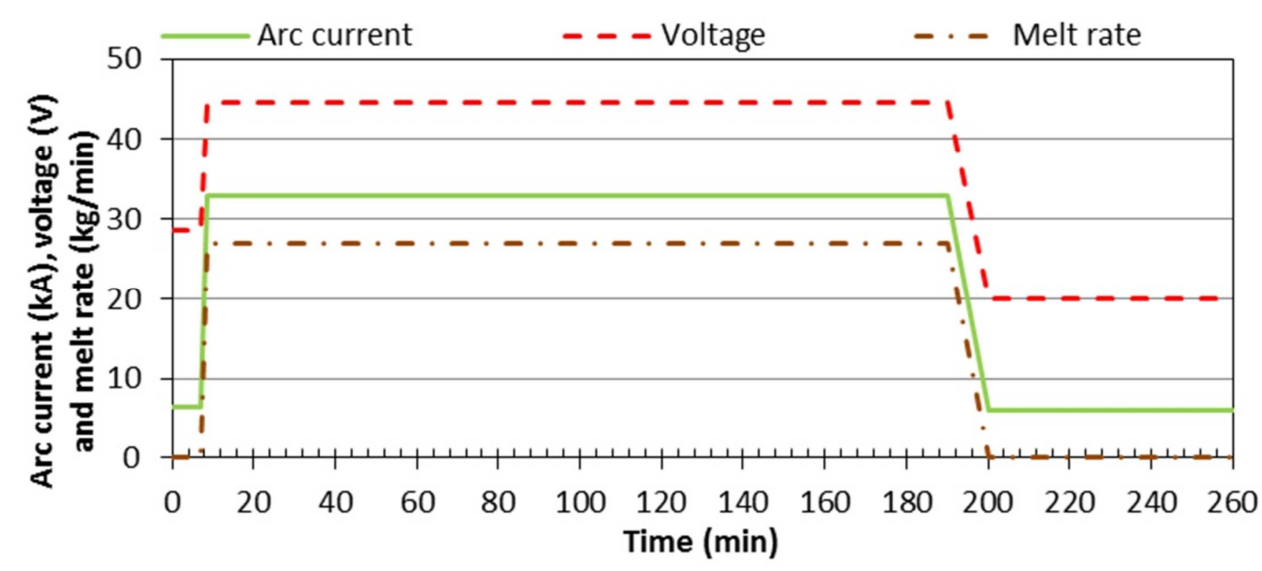

Figure 9: Operating parameters of the simulated melt. 
We first analyze the effect of the radiation modelling method on the simulation results during

381 the first sequence of the melt. Figures 10 to 12 present at a given moment during this sequence, respectively the ingot temperature field, liquid fraction map and stream function of the liquid metal motion in the molten pool.
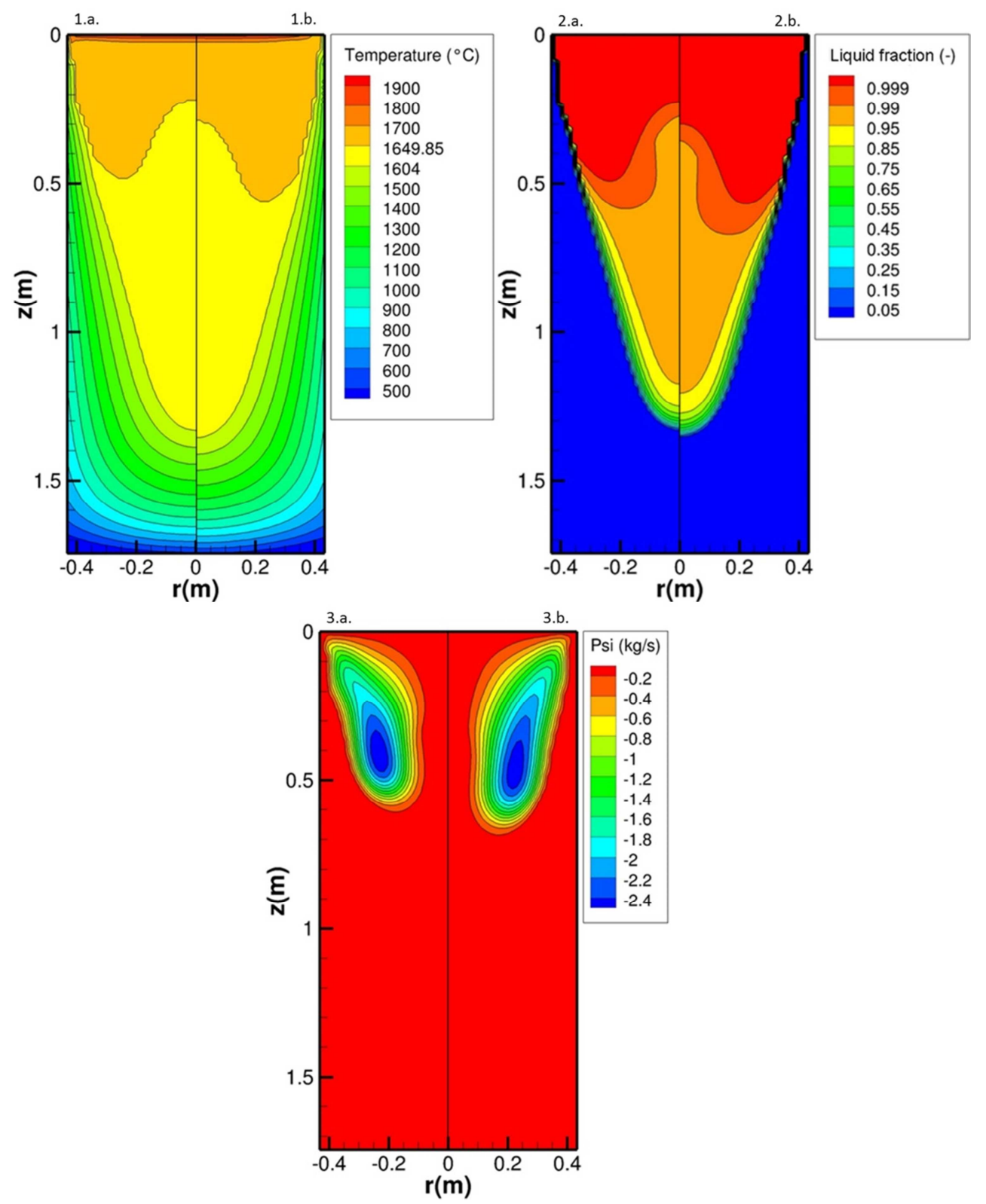

Figure 10: (1) Ingot temperature field, (2) liquid fraction map and (3) stream function with the (a) simplified and (b) advanced radiation models. 
In this figure, we observe only small differences between the two models. Use of the advanced radiation model predicts similar temperature at the ingot top (with a deviation of a few percent) but induce a notable different temperature field in the ingot, with a deeper pool. This result is related to the lower power radiated energy at the ingot top in the case of the advanced model ( $86.8 \mathrm{~kW}$ versus $88.6 \mathrm{~kW}$ with the simplified model) and the modification of the distribution of this radiation flux. The advanced model induces higher radiation at the ingot edge, as compared to the simplified model which causes locally a slightly inferior temperature. This decrease in the temperature results in a net diminution (by about 5\%) of the energy lost at the mold wall.

The differences between the results obtained using the two radiation models tend to be more important during the hot-topping stage. This is due to the much smaller energy provided by the arc during this sequence, resulting in a more important contribution of the thermal radiation in the energy balance at the ingot top. These differences are illustrated in Figure 11, which shows the evolution of the ingot temperature field during the hot-topping, predicted by both radiation models. Figure 12 presents the local solidification time in the ingot at the end of the melt. The area with a local solidification time equal to 0 corresponds to the non-fully solidified ingot (i.e. liquid pool and mushy zone). Again, the model with a more detailed description of radiative heat exchanges leads to a deeper mushy zone and a modification in the liquid pool shape. In particular the detailed model leads to lower temperature values at the 410 ingot top surface near the crucible wall. The increase in the radiation predicted with the advanced model is the cause of an earlier solidification near the ingot edge. Furthermore, it leads to a slightly lower of the local solidification time mostly at the ingot top. 

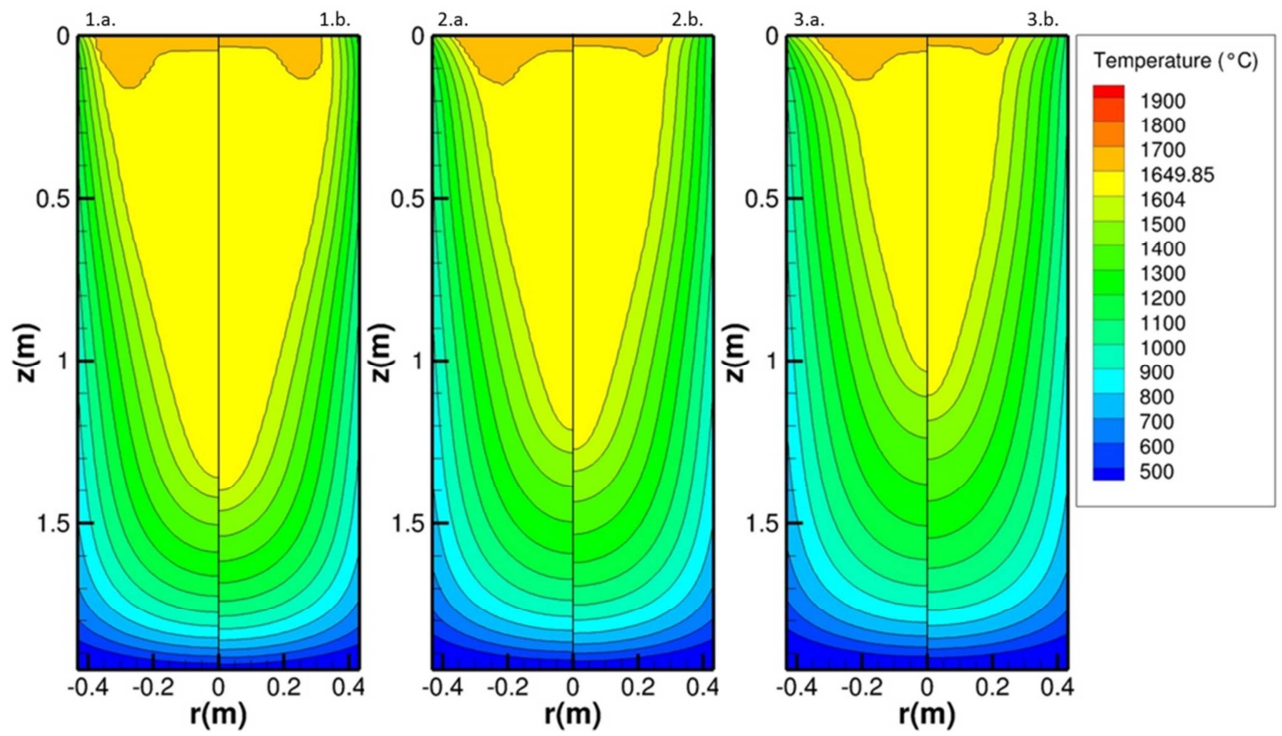

Figure 11: Ingot temperature field during the hot-topping stage computed with the (a) simplified and (b) advanced radiation models at three given moments: (1) $10 \mathrm{~min}$, (2) 30 min and (3) $50 \mathrm{~min}$ after the beginning of the hot-topping stage.

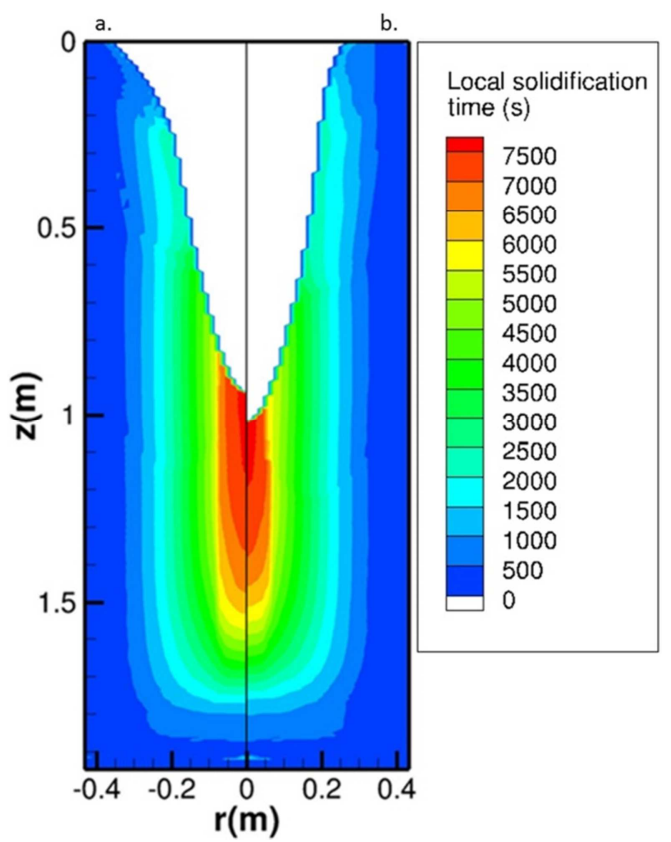

Figure 12: Computed local solidification time in the fully solidified ingot with the (a) simplified and (b) advanced radiation models at the end of the melt.

\section{Conclusion}

A numerical investigation of the ingot top thermal radiation in the vacuum arc remelting process was undertaken. Two different approaches have been compared for the calculation of 
the radiation emitted at the ingot top, namely a crude approach that considers only the radiative heat transfer between the ingot and the electrode tip, with a simplified representation of the electrode geometry, and a more detailed approach based on the radiosity method and taking into account all radiative exchanges between the ingot, electrode and crucible wall. In both cases, all surfaces are assumed as diffusive grey ones and the arc is neglected. This second approach relies on some approximations for the evaluation of non-tabulated view factors. Yet, it was verified from Monte-Carlo ray tracing simulations that those approximations have a negligible influence on the computed ingot top radiation. Results of the detailed radiation model revealed that the ingot top radiation is heavily dependent on the arc gap length and the electrode radius. By contrast, it is almost independent on the electrode height. Finally, the two proposed radiation models were implemented within a numerical code that simulates the growth and solidification of a VAR ingot. In the case of a Ti-6-4 melt, we have shown that the use of the detailed radiation model has a slight effect on the ingot simulation results when compared to these obtained with the crude radiation modelling approach, especially during the hot-topping stage. The replacement of the radiation model leads to a modification, among others, the ingot energy balance, of the ingot temperature field, the liquid pool shape and depth and the local solidification time. A precise modeling of the ingot solidification in industrial cases justifies the interest of using a detailed radiation modelling approach that considers all radiative surfaces present above the ingot top, as proposed in the present work.

1. L.A. Bertram, R.S. Minisandram and K.O. Yu: Modeling for casting and solidification processing, $1^{\text {st }}$, Marcel Dekker Inc., New York, NY, 2002. pp. 565-612.

2. A. Jardy and D. Ablitzer: Rare Met. Mater. Eng., 2006, 35, vol. 1, pp.119-122. 
451

452

453

454

455

456

457

458

459

460

461

462

463

464

465

466

467

468

469

470

471

472

473

474

475

3. K.M. Kelkar, S.V. Patankar, A. Mitchell, O. Kanou, N. Fukada and K. Suzuki: World Conf. Titanium, $11^{\text {th }}$, Kyoto, Japan, June, 3-7, The Japan Institute of Metal, Sendai, 2007,pp. 12791282.

4. K. Pericleous, G. Djambazov, M. Ward, L. Yuan and P.D. Lee: Metall. Mater. Trans. A, 2013, vol. 44, no.12, pp. 5365-5376.

5. A.S. Ballantyne: Proc. 2013 Int. Symp. on Liquid Metal Processing and Casting, Austin, TX, Sept. 22-25 2013, M.J.M. Krane, A. Jardy, R.L. Williamson, J.J. Beaman, 2013, pp. 253259.

6. A. Anders and S. Anders: J. Phys. D: Appl. Phys, 1991, vol. 24, pp. 1986-1992.

7. A.S. Ballantyne: Proc. 2015 Int. Symp. on Liquid Metal Processing and Casting, Leoben, Austria, Sept. 20-24, 2015, A. Kharicha, R.M. Ward, H. Holzgruber, M. Wu, 2015, pp. 244254.

8. J. Sucec: Heat transfer, $1^{\text {st }}$ ed., Simon \& Schuster, New York, NY, 1975, 604 pp.

9. H. Leuenberger and R.A. Person: Am. Soc. Mech. Eng., 1956, 56-A-144.

10. A.J. Buschman and C.M. Pittman: NASA, 1961, NASA-TN D-944.

11. H. Brockmann: Int. J. Heat Mass Transf., 1994, vol. 37, no. 7, pp. 1095-1100.

12. H.W. Jensen, J. Arvo, P. Dutre, A. Keller, A. Owen, M. Pharr and P. Shirley: Monte Carlo Ray Tracing, 2003, http://www.cs.odu.edu/ yaohang/cs714814/Assg/raytracing.pdf.

13. J.J. Valencia and P.N. Quested: ASM Handbook, 2008, vol. 15, pp. 468-481.

14.M. Boivineau, C. Cagran, D. Doytier, V. Eyraud, M.-H. Nadal, B. Wilthan and G. Pottlacher: Int. J. of Thermophys., 2006, vol. 27, no.2, pp. 507-529.

15. A. Jardy and D. Ablitzer: Mater. Sci. Technol., 2009, vol. 25, pp. 163-69.

16. A. Patel, D.W. Tripp and D. Fiore: Proc. 2013 Int. Symp. on Liquid Metal Processing and Casting, Austin, TX, Sept. 22-25 2013, M.J.M. Krane, A. Jardy, R.L. Williamson, J.J. Beaman, 2013, pp. 241-244. 
Tables

\begin{tabular}{|c|c|c|}
\cline { 2 - 3 } \multicolumn{1}{c|}{} & $\begin{array}{c}\text { Approximate } \\
\text { method }\end{array}$ & $\begin{array}{c}\text { Monte-Carlo } \\
\text { method }\end{array}$ \\
\hline $\mathbf{F}_{\mathbf{1 , 3}}$ & 0.01718328 & 0.03141745 \\
\hline $\mathbf{F}_{\mathbf{f} \mathbf{2} \mathbf{1}}$ & 0.16533963 & 0.13567865 \\
\hline
\end{tabular}

478

479

480

481

482

\begin{tabular}{|c|c|}
\cline { 2 - 2 } \multicolumn{1}{c|}{} & Emissivity \\
\hline Crucible & 0.8 \\
\hline Liquid metal & 0.428 \\
\hline Solid metal & 0.58 \\
\hline
\end{tabular}

Table 2: Emissivity values used in the simulations. approximate method and the Monte-Carlo method. Calculations performed for an ingot radius of $0.432 \mathrm{~m}$, an electrode radius of $0.381 \mathrm{~m}$, an electrode height of $0.05 \mathrm{~m}$ and an arc gap of $0.1 \mathrm{~m}$.

\begin{tabular}{|c|c|c|}
\hline Surface & Temperature & Comments \\
\hline $\mathbf{1}$ & $293.15 \mathrm{~K}$ & Ambient temperature \\
\hline $\mathbf{2}$ & $1923 \mathrm{~K}$ & Liquidus temperature \\
\hline $\mathbf{3}$ & $293.15 \mathrm{~K}$ & Ambient temperature \\
\hline $\mathbf{c e n}_{\mathbf{i}}$ & $2023 \mathrm{~K}$ & Liquidus temperature + superheat of $100 \mathrm{~K}$ \\
\hline int $_{\mathbf{i}}$ & $2023 \mathrm{~K}$ & Liquidus temperature + superheat of 100 K \\
\hline $\mathbf{e x t}_{\mathbf{i}}$ & $2023 \mathrm{~K}$ & Ambient temperature \\
\hline ver $_{\mathbf{i}}$ & Linear decrease of the temperature from the liquidus temperature (bottom) to \\
\hline $\mathbf{f 0}$ & $293.15 \mathrm{~K} \quad$ the ambient temperature (top) \\
\hline $\mathbf{f 1}$ & $\varnothing$ & \\
\hline $\mathbf{f 2}$ & $\varnothing$ & \\
\hline
\end{tabular}

Table 3: Temperatures of all surfaces used in the simulations.

Figure 1: VAR process schematic representation.

Figure 2: Top furnace schematic representation in the radiation model.

Figure 3: View factor between a surface element at the ingot top and the bottom surface of the electrode. Comparison between results obtained with tabulated formulas and results 
determined with the Monte-Carlo method. Calculations performed for an ingot radius of 0.432 $\mathrm{m}$, an electrode radius of $0.381 \mathrm{~m}$ and an arc gap of $0.05 \mathrm{~m}$.

Figure 4: View factor from surface elements of the ingot top toward surface 3 and surface $\mathrm{f} 2$ obtained using the approximate method and the Monte-Carlo method. Calculations performed for an ingot radius of $0.432 \mathrm{~m}$, an electrode radius of $0.381 \mathrm{~m}$, an electrode height of $0.5 \mathrm{~m}$ and an arc gap of $0.05 \mathrm{~m}$.

Figure 5: Relative difference in the heat flux density radiated at the ingot top depending on the calculation method (approximate and Monte-Carlo methods) of the non-tabulated view factors for an electrode height of $0.05 \mathrm{~m}$.

Figure 6: Ingot top radiation for different arc gaps. Calculations performed for an ingot radius of $0.432 \mathrm{~m}$, an electrode radius of $0.381 \mathrm{~m}$ and an electrode height of $1.5 \mathrm{~m}$.

Figure 7: Ingot top radiation for different electrode radii. Calculations performed for an ingot radius of $0.432 \mathrm{~m}$, an electrode height of $1.5 \mathrm{~m}$ and an arc gap of $0.05 \mathrm{~m}$.

Figure 8: Ingot top radiation for different electrode heights. Calculations performed for an ingot radius of $0.432 \mathrm{~m}$, an electrode radius of $0.381 \mathrm{~m}$ and an arc gap of $0.05 \mathrm{~m}$.

Figure 9: Operating parameters of the simulated melt.

Figure 10: (1) Ingot temperature field, (2) liquid fraction map and (3) stream function map in the molten pool at a given time during the first sequence of the melt, computed with the (a) simplified and (b) advanced radiation models.

Figure 11: Ingot temperature field during the hot-topping stage computed with the (a) simplified and (b) advanced radiation models at three given moments: (1) $10 \mathrm{~min}$, (2) $30 \mathrm{~min}$ and (3) 50 min after the beginning of the hot-topping stage.

Figure 12: Computed local solidification time in the fully solidified ingot with the (a) simplified and (b) advanced radiation models at the end of the melt. 